\title{
A STUDY OF THE LOCAL COMPONENTS OF THE HECKE ALGEBRA mod $l$
}

\author{
BY
}

NAOMI JOCHNOWITZ

\begin{abstract}
We use information about modular forms mod $l$ to study the local structure of the Hecke ring. In particular, we find nontrivial lower bounds for the dimensions of the Zariski tangent spaces of the local components of the Hecke ring $\bmod l$. These results suggest that the local components of the Hecke ring $\bmod l$ are more complex than originally expected. We also investigate the inverse limits of the Hecke rings of weight $k \bmod l$ as $k$ varies within a fixed congruence class $\bmod l-1$.

As an immediate corollary to some of the above results, we show that when $k$ is sufficiently large, an arbitrary prime $/$ must divide the index of the classical Hecke ring $\mathbf{T}_{k}$ in the ring of integers of $\mathbf{T}_{k} \otimes \mathbf{Q}$.
\end{abstract}

1. Introduction. It has been shown by Atkin, Tate, and Serre that although there are an infinite number of systems of eigenvalues for the Hecke operators in characteristic zero, there are only a finite number mod $l$. The proofs of Tate and Serre appear in a modified and generalized form in [2]. Moreover, using this result, it is shown in [2] that the power of $l$ which divides the discriminant of the classical Hecke algebra of weight $k$ grows linearly with $k$.

On close examination of Tate and Serre's proof, one sees that it implies more than the result about a finite number of systems of eigenvalues. Indeed, it gives specific information about the structure of the space of modular forms mod $l$ which can be translated into results about the local algebra of the Hecke ring.

For much of this paper we will borrow only one elementary lemma (Lemma 3.4) from Tate and Serre's proof. By combining this lemma with basic properties of some well-known operators on the space of modular forms $\bmod l$, we obtain significant information about the structure of the Hecke ring $\bmod l$ and also an interesting result about the structure of the classical Hecke ring.

For the purposes of simplicity, we limit ourselves to the case of the full modular group, although many of our results generalize to higher levels. Unless otherwise stated, we let $l$ be a prime not equal to 2 or 3 .

The Hecke ring mod $l$ of weight $k$, when tensored with the algebraic closure, can be written as a direct sum of local components, each component corresponding to a specific system of eigenvalues $\bmod l$. Moreover, we see in $\$ 3$, that when $k$ is sufficiently large, the operator $U=T_{l}$ will have a nilpotent image in some of these components. We call such components $U$-nilpotent and show that the non- $U$ nilpotent components are in a certain sense trivial.

Received by the editors July 18,1979 and, in revised form, January $22,1981$.

1980 Mathematics Subject Classification. Primary 10D12, 10D23.

(C) 1982 American Mathematical Society 0002-9947/81/0000-1033/\$04.75 
One goal of this paper is to obtain a partial description of the $U$-nilpotent components of the Hecke ring mod $l$. For example, in $\$ 4$, we examine the Zariski tangent dimensions ${ }^{1}$ of the $U$-nilpotent components of weight $k$. Specifically, we find positive integers $B_{1}, B_{2}$, such that if $k \geqslant B_{1}$, the dimension of the Zariski tangent space of at least one $U$-nilpotent component is greater than or equal to two, and if $k \geqslant B_{2}$ the same is true for all $U$-nilpotent components. Moreover, in $\S 8$ we show that when $k$ is sufficiently large, with the possible exception of a particular class of components, the Zariski tangent dimension of all $U$-nilpotent components is at least three. These results imply that the local components of the Hecke ring mod $l$ are more complex than originally expected.

As an immediate corollary of the first result mentioned above, we prove a result about the classical Hecke ring $\mathbf{T}_{k}$. In particular, we show that any prime $l$ will divide the index of $\mathbf{T}_{k}$ in the ring of integers of $\mathbf{T}_{k} \otimes \mathbf{Q}$ when the weight is sufficiently large.

It is interesting to observe what happens to the structure of the local components of the Hecke ring mod $l$ as the weight $k$ approaches infinity. In order to do this, we select a system of eigenvalues mod $l$ and consider the corresponding local components of weight $k$, as $k$ varies within a fixed congruence class $\bmod l-1$. These components form a surjective system of local rings, and in $\$ 6$ we study the inverse limit of such a system in the $U$-nilpotent (i.e. nontrivial) case. In particular, we show that these inverse limits are isomorphic to power series rings in the variable $U$ over infinite-dimensional $\mathbf{F}_{r}$-algebras. It follows easily that in the case where the Zariski tangent dimension equals two, the inverse limit must be isomorphic to a power series ring in two variables over $\mathbf{F}_{l}$.

Many unanswered questions remain and, despite all of the information acquired, the exact structure of the Hecke ring remains elusive, even in characteristic $l$. For example, although we have found a lower bound for the Zariski tangent dimension of the local components, the following question is still an open one. As $k$ approaches infinity do these dimensions become arbitrarily large or do they remain bounded? In particular, are they ever greater than three?

The author wishes to thank John Tate and David Kazhdan for their advice and concern, and especially Barry Mazur for his help and encouragement.

2. Modular forms mod $l$. In this section we briefly review some of the basic facts about modular forms mod $l$. Let

$$
M_{k}=\left\{f=\sum a_{n} q^{n} \mid f \in A(k), a_{n} \in \mathbf{Z}\right\}
$$

be the lattice of all modular forms of weight $k$ for the full modular group whose $q$-expansions have integer coefficients. Define $\tilde{M}_{k}$, the space of modular forms mod $l$ of weight $k$, to be the $\mathbf{F}_{\digamma}$-vector space

$$
\tilde{M}_{k}=\left\{\tilde{f}=\sum \tilde{a}_{n} q^{n} \mid f=\sum a_{n} q^{n} \in M_{k}\right\} \subseteq \mathbf{F}_{/}[[q]],
$$

\footnotetext{
${ }^{1}$ Definition 1.1. If $A$ is a local ring with maximal ideal $m$, then the Zariski tangent space of $A$ is defined to be the dual of the space $m / m^{2}$ when considered as an $A / m$ vector space. In particular, the Zariski tangent dimension equals the $A / m$ dimension of $\mathrm{m} / \mathrm{m}^{2}$.
} 
where $\tilde{a}_{n}$ denotes the reduction of $a_{n} \bmod l$. We also define the space of all modular forms $\bmod l$ to be the sum of the spaces $\tilde{M}_{k}$ and denote it by $\tilde{M}$.

Swinnerton-Dyer and Serre ([8] and [6]) showed that $\tilde{M}_{k} \subseteq \tilde{M}_{k+l-1}$ and that $\tilde{M}_{k}$ has a nontrivial intersection with $\tilde{M}_{k^{\prime}}$ only when $k \equiv k^{\prime}(\bmod l-1)$. The concept of the weight of a modular form is thus no longer well defined $\bmod l$, and we replace it by the more useful concept of filtration.

Definition 2.1. The filtration of a modular form $f \in \tilde{M}_{k}$ is the quantity $w(f)=$ $\inf \left\{j \mid f \in \tilde{M}_{j}\right\}$.

The Hecke operators induce an action on the space $\tilde{M}_{k}$. Of special interest is the operator $T_{l}$ which coincides mod $l$ with Atkin's $U_{l}$ operator and is therefore denoted by $U$. In terms of the $q$-expansions of a modular form $\bmod l$ of weight $k$, we have the following formulae.

FORMULAE 2.1. (a) $U: \sum a_{n} q^{n} \mapsto \sum a_{n l} q^{n}$.

(b) $T_{p}: \sum a_{n} q^{n} \mapsto \sum a_{n p} q^{n}+p^{k-1} \sum a_{n} q^{n p}$ if $p \neq l$.

In order to investigate the structure of the Hecke ring, we introduce two other operators on the space of modular forms mod $l$.

(a) $V: \sum a_{n} q^{n} \mapsto \sum a_{n} q^{n l}$.

(b) $\theta=q(d / d q): \sum a_{n} q^{n} \mapsto \sum n a_{n} q^{n}$.

The map $\theta^{l-1}$ is of special importance. It takes the modular form mod $l$ with $q$-expansion $\sum_{n=0}^{\infty} a_{n} q^{n}$ to the modular form with $q$-expansion $\sum_{(n, l)=1} a_{n} q^{n}$. In particular, $\theta^{l-1}$ acts as the identity when restricted to the kernel of $U$.

The following facts describe some relations between the operators $V, \theta, U$, and $T_{p}$. They are easily verified from the above formulae and play a significant role in the proofs of this paper. Note that throughout this paper, we write all operators on the right.

FACT 2.2. (a) $V$ and $T_{p}$ commute if $p \neq i$.

(b) $V \circ U=$ Identity.

(c) $\theta \circ T_{p}=p T_{p} \circ \theta$. In particular, if $p \neq l, T_{p}$ commutes with $\theta^{l-1}$.

(d) image $\theta=\operatorname{kernel} U$.

(e) kernel $\theta=$ image $V$.

3. The local components of $R_{k} \otimes \overline{\mathbf{F}}_{l}$. We define $R_{k}$, the Hecke ring mod $l$ of weight $k$, to be the subring of End $\mathbf{F}_{l} \tilde{M}_{k}$ generated by the Hecke operators. If $\mathbf{T}_{k}$ denotes the classical Hecke ring of weight $k$ (see §5), then $R_{k} \approx \mathbf{T}_{k} / l \mathbf{T}_{k}$ [5].

Each maximal ideal of $R_{k} \otimes \overline{\mathbf{F}}_{l}$ corresponds to a unique system of eigenvalues for the Hecke operators of weight $k \bmod l$. Such an ideal is generated by the operators $T_{p}-\lambda_{p}$ where $\left\{\lambda_{p}\right\}$ is the corresponding system of eigenvalues. Since $R_{k} \otimes \overline{\mathbf{F}}_{l}$ is an Artin ring it can be written uniquely as a direct sum of local components. Each such component corresponds to a unique maximal ideal of $R_{k} \otimes \overline{\mathbf{F}}_{l}$ and, hence, to a unique system of eigenvalues.

Definition 3.1. Let $A_{k}$ be a local component of $R_{k} \otimes \overline{\mathbf{F}}_{l}, \mathfrak{m}$ the associated maximal ideal, and $\left\{\lambda_{p}\right\}$ the corresponding system of eigenvalues. We define $S_{k}$, the generalized eigenspace associated to $A_{k}$, to be the set of those forms in $\tilde{M}_{k} \otimes \overline{\mathbf{F}}_{l}$ which are annihilated by some power of the ideal $\mathrm{m}$. Equivalently, $S_{k}$ is the set of all $g \in \tilde{M}_{k} \otimes \overline{\mathbf{F}}_{l}$ which are annihilated by some power of the operators $T_{p}-\lambda_{p}$ for all $p$. 
Any local component $A_{k}$ acts faithfully on its generalized eigenspace $S_{k}$ and annihilates the other generalized eigenspaces of weight $k$. Moreover the space

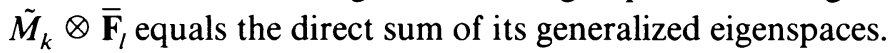

By abuse of notation, we use the letters $T_{p}$ or $U$ to denote the images of the operators $T_{p}$ or $U$ in the local component $A_{k}$. Then a local component is called $U$-nilpotent if the operator $U$ is nilpotent in it. We now show that the non- $U$ nilpotent components are trivial in the sense that their associated generalized eigenspaces contain only elements of low filtration.

Lemma 3.2. Let $S_{k}$ be a generalized eigenspace of $\tilde{M}_{k} \otimes \overline{\mathbf{F}}_{l}$ which contains a form of filtration greater than $l+1$. Then the associated local component of $R_{k} \otimes \overline{\mathbf{F}}_{l}$ is U-nilpotent.

Proof. Suppose that the lemma is false. Then $U$ is a unit in this local component and therefore acts bijectively on $S_{k}$.

Choose $h \in S_{k}$ of maximal filtration. There exists $g \in S_{k}$ such that $g \mid U=h$. By the definition of filtration, we clearly have $w(g) \geqslant w(g \mid U)>l+1$. But Lemma 1.9 of [2] states that if $w(g)>l+1$, then applying $U$ decreases the filtration. Therefore, $w(h)$ is strictly less than $w(g)$ which contradicts our choice of $h$. Q.E.D.

COROLlary 3.3. If $k$ is sufficiently large, $R_{k} \otimes \overline{\mathbf{F}}_{l}$ contains at least one U-nilpotent component.

Proof. Immediate.

Let $W_{k}$ be the vector space $\left(\tilde{M}_{k} \otimes \overline{\mathbf{F}}_{l}\right) /\left(\tilde{M}_{k-l+1} \otimes \overline{\mathbf{F}}_{l}\right)$. In other words, $W_{k}$ is the quotient space of forms of weight $k$ with coefficients in $\overline{\mathbf{F}}_{l}$ modulo the subvector space of all such forms of lower filtration.

Let $R$ be the subring of $\operatorname{End}_{\mathbf{F}_{l}} \tilde{M}$ generated by the Hecke operators. We use the term Hecke module to mean a module over the ring $R$. Since the Hecke operators clearly induce an action on the quotient space $W_{k}$, we may view $W_{k}$ as a Hecke module.

The following lemma plays an important role in Tate and Serre's proof about the finite number of systems of eigenvalues mod $l$. It also plays a critical role in several of the proofs of this paper. Its original proof is due to Serre.

LeMma 3.4. If $k \geqslant l+1$, then $W_{k} \approx W_{k l-l^{2}+1}$ as a Hecke module.

Proof. See Case 2 of the proof of Theorem 2.4 in [2].

4. The Zariski tangent dimension. In this section we show that if $k$ is sufficiently large, the dimension of the Zariski tangent space (Definition 1.2) of at least one of the $U$-nilpotent components of $R_{k} \otimes \overline{\mathbf{F}}_{l}$ will be greater than or equal to two, and when $k$ is even larger, the analogous statement is true about all $U$-nilpotent components.

Recall the operators $\theta$ and $V$ introduced in $\$ 2$. The following lemma summarizes the effect of these operators on the filtration of a modular form $\bmod l$. 
LEMMA 4.1. (a) $w(f \mid V)=\operatorname{lw}(f)$.

(b) The operator $\theta$ maps $\tilde{M}_{k}$ to $\tilde{M}_{k+1+1}$. Moreover, $w(f \mid \theta) \leqslant w(f)+l+1$, with equality if and only if $l+w(f)$.

Proof. Part (a) follows from Lemma 1.b of $\$ 2.2$ of [7], and (b) is proven in [6] and [8]. Q.E.D.

LEMMA 4.2. Let $S_{k}$ be a generalized eigenspace of weight $k$ which contains an element of filtration $j$ where $l+1<j \leqslant k / l$. Then the Zariski tangent dimension of the corresponding local component of $R_{k} \otimes \overline{\mathbf{F}}_{l}$ is greater than or equal to two.

Proof. Lemma 3.2 implies that we are in the $U$-nilpotent case. Let $g \in S_{k}$ be a simultaneous eigenvector of filtration less than or equal to $j$. The proof proceeds in two steps. We first show that $S_{k}$ contains a form $h \in$ Kernel $U$ which is not a scalar multiple of $g$, and then show that this implies that the corresponding Zariski tangent dimension is at least two.

Step 1. Lemma 3.4 implies that $S_{k}$ contains some element $h_{1}$ of filtration $j l-l^{2}+1$. Let $h=h_{1} \mid \theta^{l-1}$. Then by Lemma 4.1, $w(h)=j l$. Since $k \geqslant j l$ and $S_{k}$ is $U$-nilpotent, it follows that $h$ belongs to $S_{k}$. Moreover, $h$ is clearly annihilated by $U$, but by comparing filtrations, one sees immediately that $h$ is not a scalar multiple of g.

Step 2. Let $A_{k}$ be the corresponding local component of $R_{k} \otimes \overline{\mathbf{F}}_{l}$ and let $\mathrm{m}$ be its maximal ideal. Since $A_{k}$ is $U$-nilpotent, $U \in \mathfrak{m}$. However, $U$ is not an element of $\mathrm{m}^{2}$ since $g \mid V \in S_{k}$ is annihilated by the ideal $\mathrm{m}^{2}$ but is not annihilated by $U$.

Suppose now that the Zariski tangent dimension of $A_{k}$ is less than or equal to one. The above paragraph then implies that the ideal $m$ is generated by $U$. Therefore, the form $h$, introduced above, is annihilated by $\mathfrak{m}$, and hence is a simultaneous eigenvector with the same system of eigenvalues as $g$. Because of the relationship between eigenvalues and coefficients of $q$-expansions, this contradicts the fact that $h$ is not a scalar multiple of $g$. Q.E.D.

THEOREM 4.3. If $l \geqslant 13$, then whenever $k \geqslant 2 l^{2}$, the Zariski tangent dimension of at least one $U$-nilpotent component of $R_{k} \otimes \overline{\mathbf{F}}_{l}$ will be greater than or equal to two. If $l=5$, the result is true whenever $k \geqslant 3 l^{2}+3 l$, and if $l=7$ or 11 , whenever $k \geqslant 3 l^{2}-l$.

PROOF. The cases where $l<13$ are exceptional since they are the only cases for which there do not exist modular forms of every possible filtration.

The theorem follows as an immediate corollary to Lemma 4.2 after noting that when $k$ is as above, some generalized eigenspace of weight $k$ must contain an element $h$ of filtration

$$
l+1<w(h) \leqslant \begin{cases}2 l & \text { if } l \geqslant 13 \\ 3 l-1 & \text { if } l=7 \text { or } 11 \\ 3 l+3 & \text { if } l=5 . \quad \text { Q.E.D. }\end{cases}
$$

LEMMA 4.4. Any generalized eigenspace contains a simultaneous eigenform of filtration less than or equal to $l^{2}+l$. In particular, there are only a finite number of systems of eigenvalues mod $l$. 
Proof. We have already seen (Lemma 3.2) that all non- $U$-nilpotent generalized eigenspaces contain only forms of filtration less than or equal to $l+1$. Moreover, Theorem 4.1 of [2] (whose proof is due to Tate and Serre) implies that any eigenform in the $U$-nilpotent case can be written as $f \mid \theta^{a}$ where $a \leqslant l-1$ and $w(f) \leqslant l+1$. Lemma 4.1 concludes the proof. Q.E.D.

THEOREM 4.5. If $k \geqslant l^{3}+l^{2}$, the Zariski tangent dimension of all U-nilpotent components of $R_{k} \otimes \overline{\mathbf{F}}_{l}$ will be greater than or equal to two.

Proof. By Lemma 4.2, it suffices to show that when $k \geqslant l^{3}+l^{2}$, any $U$-nilpotent generalized eigenspace contains an element of filtration $j$ where $l+1<j \leqslant l^{2}+l$.

The previous lemma states that any such generalized eigenspace contains a form $g$ of filtration less than or equal to $l^{2}+l$. Moreover, if $w(g) \leqslant l+1$, then $l+1<$ $w(g \mid V) \leqslant l^{2}+l$ by Lemma 4.1. Q.E.D.

5. Applications to the classical case. As a corollary to Theorem 4.3 , we obtain the following result about the classical Hecke ring. Let $A(k)$ be the space of automorphic forms of weight $k$ for the full modular group, and let $\mathbf{T}_{k}$ be the commutative subring of End $A(k)$ generated by the Hecke operators. The ring $\mathbf{T}_{k}$ is called the classical Hecke ring.

It is well known that $\mathbf{T}_{k} \otimes \mathbf{Q}$ is isomorphic to a direct product of totally real number fields, and that $\mathbf{T}_{k}$ is a subring of finite index in the ring of integers $\theta_{k}$ of $\mathbf{T}_{k} \otimes \mathbf{Q}$. The index of $\mathbf{T}_{k}$ in $\vartheta_{k}$ has been computed when $k$ is relatively small, but it is natural to ask what happens to this index as $k$ approaches infinity.

THEOREM 5.1. Let $\mathbf{T}_{k}$ and $\theta_{k}$ be as above. Then if $l \geqslant 13, l$ divides the index $\left[{ }^{0}, \mathbf{T}_{k}\right]$ whenever $k \geqslant 2 l^{2}$. If $l=7$ or $11, l$ divides the index whenever $k \geqslant 3 l^{2}-l$, and if $l=5$, whenever $k \geqslant 3 l^{2}+3 l$.

Proof. Suppose that $l$ does not divide the index $\left[\theta_{k}: \mathbf{T}_{k}\right]$. Then multiplication by $l$ is an isomorphism of the additive group $\theta_{k} / \mathbf{T}_{k}$. Therefore, $\theta_{k}=\mathbf{T}_{k}+l \theta_{k}$ which implies that the canonical map $\phi_{k}: \mathbf{T}_{k} / l \mathbf{T}_{k} \rightarrow \hat{C}_{k} / l \Theta_{k}$ is a surjection. Since $\mathbf{T}_{k} / l \mathbf{T}_{k}$ and $\varepsilon_{k} / l \hat{\theta}_{k}$ have equal dimensions as $\mathbf{F}_{\digamma}$-vector spaces, $\phi_{k}$ is in fact an isomorphism.

Since $\theta_{k}$ is a finite direct product of Dedekind domains, $\theta_{k} / l \theta_{k}$ is a principal ideal ring. The above isomorphism thus implies that $\mathbf{T}_{k} / l \mathbf{T}_{k}$ is also a principal ideal ring.

But we have already seen that $\mathbf{T}_{k} / l \mathbf{T}_{k}$ is isomorphic to $R_{k}$. It follows that the Zariski tangent spaces of all of the local components of $R_{k}$, and hence of $R_{k} \otimes \overline{\mathbf{F}}_{l}$, have dimensions less than or equal to one.

Theorem 4.3 then implies that if $l=5, k<3 l^{2}+3 l$, if $l=7$ or $11, k<3 l^{2}-l$, and if $l \geqslant 13, k<2 l^{2}$. Q.E.D.

The author has also proven that the power to which any prime $l$ divides the index $\left[\mathfrak{C}_{k}: \mathbf{T}_{k}\right]$ approaches infinity as $k$ does [1]. The proof uses results of this paper.

6. Inverse limits of $U$-nilpotent components. Since $\tilde{M}_{j} \otimes \overline{\mathbf{F}}_{l} \subseteq \tilde{M}_{j+l-1} \otimes \overline{\mathbf{F}}_{l}$, there exists a canonical surjection $R_{j+l-1} \otimes \overline{\mathbf{F}}_{l} \rightarrow R_{j} \otimes \overline{\mathbf{F}}_{l}$. Moreover, if $A_{j+l-1}$ and $A_{j}$ are local components which correspond to the same system of eigenvalues, then $A_{j+l-1}$ maps onto $A_{j}$ under the above surjection. In this way, if we fix an arbitrary system of 
eigenvalues mod $l$, we obtain a corresponding surjective system of local components, $A_{j} \leftarrow A_{j+l-1} \leftarrow A_{j+2 l-2} \leftarrow \ldots$.

By considering the inverse limits of these surjective systems, we gain information about the various rings $A_{k}$ as $k$ approaches infinity. The goal of this section is to describe these inverse limits.

The inverse limit in the non- $U$-nilpotent case is not very interesting. In fact, Lemma 3.2 implies that in such a case, $A_{j} \approx A_{j+l-1} \approx A_{j+2 l-2} \approx \ldots$ whenever $j \geqslant 4$.

Thus, from now on, any component $A_{k}$ which appears in this section will be assumed to be $U$-nilpotent. We define $C_{k}$ to be the subring of $A_{k}$ generated by the Hecke operators $T_{p}$ for $p \neq l$, and we refer to $C_{k}$ as the $U$-independent subring of $A_{k}$. Note that this terminology is somewhat deceptive. For example, under the right conditions, the operator $U$ may belong to $C_{k}$.

To each surjective system of local components $A_{k}$, there corresponds a surjective system of the subrings $C_{k}$. Let $A$ and $C$ denote the respective inverse limits of the systems

$$
A_{j} \leftarrow A_{j+l-1} \leftarrow A_{j+2 l-2} \leftarrow \ldots
$$

and

$$
C_{j} \leftarrow C_{j+l-1} \leftarrow C_{j+2 l-2} \leftarrow \ldots .
$$

We may clearly view $C$ as a subring of $A$. We denote by the letter $U$ that element of $A$ which maps onto $U$ in all of the rings $A_{k}$.

THEOREM 6.3. Keeping the above notation, the ring $A$ is isomorphic to a power series ring in the variable $U$ over the ring $C$.

Proof. A standard argument using the fact that $U$ is nilpotent in each $A_{k}$ and that each ring $C_{k}$ is an Artin ring implies that $A$ is isomorphic to a quotient of the above power series ring.

To complete the proof we must show that any operator of the form $\sum_{i=J}^{\infty} c_{i} U^{i}$, where $c_{i} \in C$ and $c_{J} \neq 0$, has a nonzero image in the rings $A_{k}$ when $k$ is sufficiently large. We do this by exhibiting a form $h$ in the extended eigenspace corresponding to $A_{k}$ which is not annihilated by $\sum_{i=J}^{\infty} c_{i} U^{i}$.

Since $c_{J} \neq 0$, then when $k$ is sufficiently large we can choose a form $g$ in the generalized eigenspace corresponding to $A_{k}$ such that $g$ is not annihilated by $c_{J}$.

By repeated application of the operator $U$ if necessary, we can adjust our function $g$ so that $g \mid c_{J}$ does not belong to the image of $V$. By Fact 2.2(e), this implies that $g\left|c_{J}\right| \theta^{l-1} \neq 0$.

Let $h=g\left|\theta^{l-1}\right| V^{J}$. One easily checks that

$$
h\left|c_{J} U^{J}=g\right| \theta^{l-1}\left|c_{J}=g\right| c_{J} \mid \theta^{l-1} \neq 0,
$$

but $h \mid c_{i} U^{i}=0$ for all $i>J$.

This implies that $h$ is not annihilated by $\sum_{i=J}^{\infty} c_{i} U^{i}$. Q.E.D.

COROLlaRy 6.4. $A$ is isomorphic to a power series ring over a ring which is not a field. 
Proof. Theorem 4.5 implies that the Zariski tangent dimension of $A$ is at least two. Q.E.D.

The ring $A$ is obviously an infinite-dimensional $\overline{\mathbf{F}}_{f}$ algebra. We now show that the same is true about the ring $C$.

Let $S_{k}$ be the generalized eigenspace corresponding to $A_{k}$, and let $S=\cup S_{k}$ be the union as $k$ varies over all integers in the above congruence class. We define the quotient space $\bar{S}=S /($ image $V \cap S$ ).

Since $C$ stabilizes the space image $V \cap S, \bar{S}$ may clearly be viewed as a $C$ module. Moreover, for any nonzero $T \in C$, there exists $g \in S$ such that $g \mid T \neq 0$. By repeated application of the operator $U$ if necessary, we can adjust our function $g$ so that $g \mid T$ is not in the image of $V$. Therefore $C$ acts faithfully on the quotient space $\bar{S}$.

Lemma 6.5. There exists a nondegenerate bilinear pairing between the $\overline{\mathbf{F}}_{\tau}$ vector spaces $\bar{S}$ and $C$ defined by $\langle\bar{f}, T\rangle=a_{1}(f \mid T)=$ the first coordinate of $f \mid T$.

Proof. If $f \in$ image $V, a_{1}(f \mid T)=0$ for all $T \in C$. Thus the above pairing is well defined. It remains to show that the kernels on the right and left are trivial.

Suppose that there exists $\bar{f} \in \bar{S}$ such that $\langle\bar{f}, T\rangle=0$ for all $T \in C$. Then, in particular, $a_{m}(f)=a_{1}\left(f \mid T_{m}\right)=\left\langle\bar{f}, T_{m}\right\rangle=0$ for all integers $m$ relatively prime to $l$. Therefore $f$ belongs to the image of $V$ and, hence, $\bar{f}=0$ in $\bar{S}$.

Suppose, on the other hand, that there exists $T_{1} \in C$ such that $\left\langle\bar{f}, T_{1}\right\rangle=0$ for all $\bar{f} \in \bar{S}$. Then

$$
0=\left\langle\bar{f} \mid T, T_{1}\right\rangle=a_{1}\left(f|T| T_{1}\right)=a_{1}\left(f\left|T_{1}\right| T\right)=\left\langle\bar{f} \mid T_{1}, T\right\rangle
$$

for all $\bar{f} \in \bar{S}$ and all $T \in C$. By the previous paragraph, this implies that $\bar{f} \mid T_{1}=0$ in $\bar{S}$ for all $\bar{f} \in \bar{S}$. Since $C$ acts faithfully on $\bar{S}, T_{1}$ must equal zero. Q.E.D.

COROllary 6.6. $C$ is infinite dimensional as an $\overline{\mathbf{F}}_{f}$ vector space.

Proof. By the previous lemma, it suffices to show that $\bar{S}$ is infinite dimensional.

The space $S$ will always contain at least one modular form $g_{0}$ of filtration $k_{0}>l+1$. (For example, if $f \in S$ has a filtration which is too small, use $f \mid V$.)

By repeated application of Lemma 3.4, we can choose modular forms $g_{n} \in S$ of filtration $k_{n}$ for all integers $n \geqslant 1$, where $k_{n}=k_{n-1} l-l^{2}+1$.

Any finite linear combination of the forms $g_{n}$ with $n \geqslant 1$ will be of filtration congruent to one mod $l$ and, hence, cannot be in the image of $V$ (Lemma 4.1). Therefore the images of the forms $g_{n}, n \geqslant 1$, are all linearly independent in the space $\bar{S}$. Q.E.D.

It is an open question whether or not there exist any $U$-nilpotent surjective systems whose inverse limits have Zariski tangent dimensions equal to two. We have already seen that these dimensions can never be less than two, and we show in $\S 8$ that in "most cases" they are greater than or equal to three. The next proposition shows that if such two-dimensional surjective systems do exist, their inverse limits are completely determined.

Proposition 6.7. Let the rings $A_{k}, A, C_{k}, C$ be as above. Suppose that $A$ has a Zariski tangent dimension of exactly two. Then $A \approx \overline{\mathbf{F}}_{l}[[X, Y]]$. 
Proof. Under the above hypotheses, Theorem 6.3 implies that the Zariski tangent dimension of $C$ equals one. Hence $C$ is isomorphic to a quotient of $\overline{\mathbf{F}}_{l}[[X]]$. The previous corollary implies that $C$ must equal the whole ring $\overline{\mathbf{F}}_{l}[[X]]$. We conclude the proof by applying Theorem 6.3 one more time. Q.E.D.

7. $\theta$-cycles. The ideas presented in this section were derived from a seminar talk by John Tate. Until now, they were unpublished.

Fact 4.1 states that $w(f \mid \theta) \leqslant w(f)+l+1$ with equality if and only if $l \nmid w(f)$. In particular, if $w(f) \equiv 1 \bmod l$, then $w\left(f \mid \theta^{A}\right)=w(f)+A(l+1)$ for all $1 \leqslant A \leqslant$ $l-1$. We call the ordered set $\left\{w\left(f \mid \theta^{A}\right) \mid A=1,2, \ldots, l-1\right\}$ the $\theta$-cycle belonging to $f$, and we represent it by the following diagram.

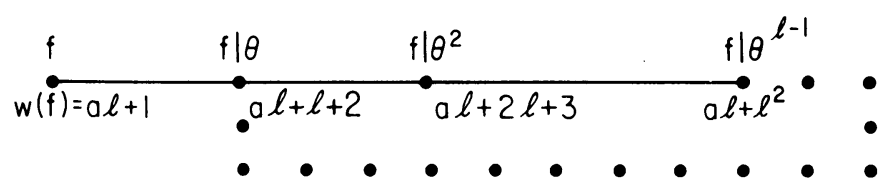

FIGURE 7.1

The dotted line here indicates that $f\left|\theta^{l}=f\right| \theta$, and hence one continues in a cycle. Since $w(f) \neq w\left(f \mid \theta^{A}\right)$ for any integer $A$, we say that $f$ is outside of its $\theta$-cycle.

We next consider $w(f) \equiv B \bmod l$ with $2 \leqslant B \leqslant l$. Then Fact 4.1 implies that $w\left(f \mid \theta^{A}\right) \leqslant w(f)+A(l+1)$. In fact, each application of $\theta$ increases the filtration by exactly $l+1$ unless the previous filtration is divisible by $l$. If the previous filtration is divisible by $l$, applying $\theta$ first adds $l+1$ to the filtration, and then subtracts off a positive integral multiple of $l-1$.

Thus if we start with a form $f_{1}$ which is in the kernel of $U$, we can schematically represent the $\theta$-cycle of $f_{1}$ as follows.

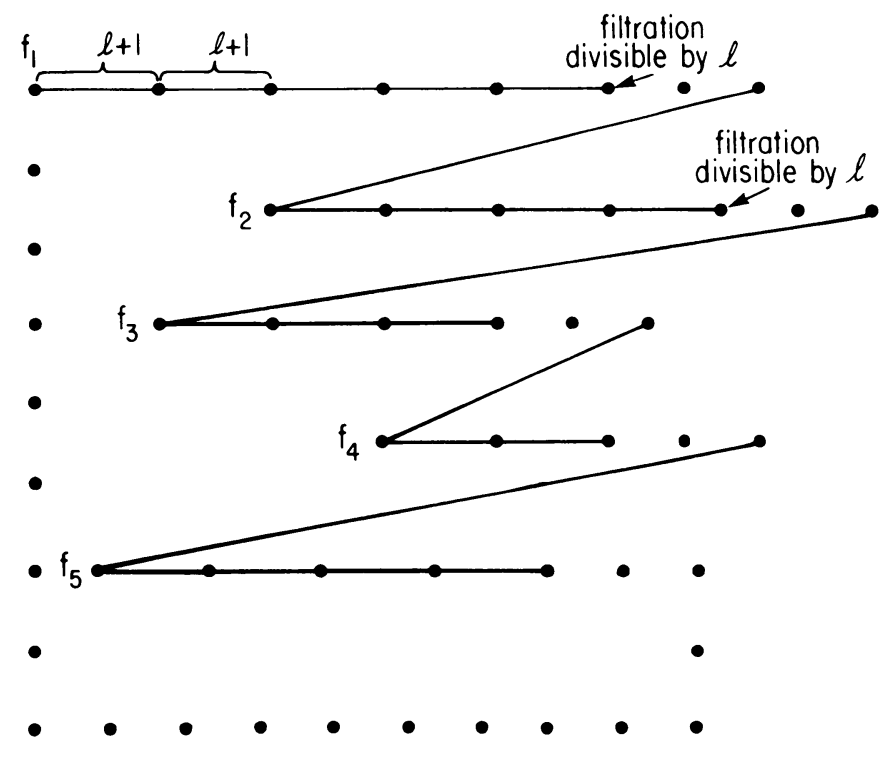

FIGURE 7.2 
The dotted line at the very end indicates that since $f_{1}$ is in the kernel of $U$, then $f_{1} \mid \theta^{1-1}=f_{1}$.

Definition 7.3. $f_{i}$ is called a low point of its $\theta$-cycle if it occurs immediately after a fall, equivalently if $f_{i}=f_{1} \mid \theta^{A}$ and $f_{1} \mid \theta^{A-1}$ has filtration divisible by $l$.

The goal of this section is to explicitly determine the $\theta$-cycle of $f_{1}$. To do so we introduce the following notation. For each low point of the $\theta$-cycle, $f_{i}$, we let $c_{i}-1$ be the number of times we add $l+1$ to $w\left(f_{i}\right)$ before reaching a filtration which is divisible by $l$, and we let $b_{i}(l-1)$ be the amount which we fall (after adding $l+1$ to the filtration) with the next application of $\theta$.

Since a complete cycle includes a total of $l-1$ applications of $\theta$, then $\Sigma_{i} c_{i}=l-1$. Since the cycle ends exactly where it begins, we note that

$$
(l+1)(l-1)=\sum_{i} c_{i}(l+1)=\sum_{i} b_{i}(l-1)
$$

and conclude that $\Sigma b_{i}=l+1$.

Moreover, when one starts at a filtration which is divisible by $l$, adds $l+1$, falls $b_{i}(l-1)$, and then adds $\left(c_{i+1}-1\right)(l+1)$, one again reaches a filtration which is divisible by $l$. Therefore,

$$
c_{i+1}+b_{i} \equiv c_{i+1}(l+1)-b_{i}(l-1) \equiv 0(\bmod l) .
$$

Since

$$
\sum_{i}\left(c_{i+1}+b_{i}\right)=\sum_{i} c_{i}+\sum_{i} b_{i}=l+1+l-1=2 l,
$$

then there are only two logical possibilities. Either there is only one fall and $c_{1}=l-1, b_{1}=l+1$, or there are two falls and $b_{1}=l-c_{2}, b_{2}=l-c_{1}$.

The first case happens if and only if $w\left(f_{1}\right) \equiv 2 \bmod l$. In the second case, $w\left(f_{1}\right) \equiv B \bmod l$ with $3 \leqslant B \leqslant l$, and one easily calculates that $c_{1}=l-B+1$, $c_{2}=B-2, b_{1}=l-B+2$, and $b_{2}=B-1$. We thus have the following pictures.

Case 1 .

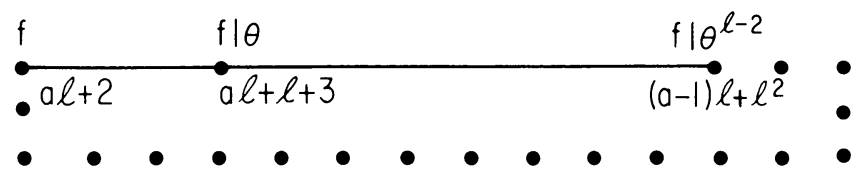

Case 2.

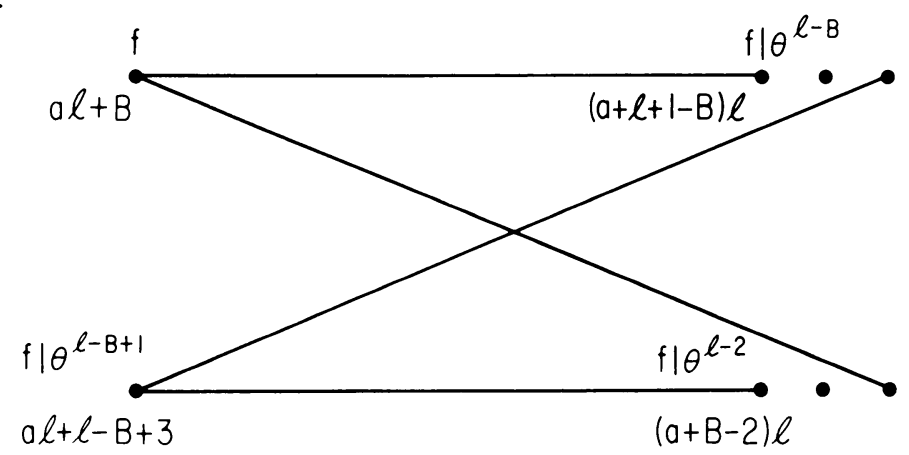

FIGURE 7.4 
Corollary 7.5. If $f$ is in the kernel of $U$ and if $l$ divides $w(f)$, then $w(f \mid \theta) \leqslant$ $w(f)-l+3$.

Proof. We know that $w(f \mid \theta)=w(f)+l+1-b(l-1)$, where $b$ is a positive integer (Lemma 4.1). The above examples show that $b$ is at least 2. Q.E.D.

We now apply the above theory to determine the $\theta$-cycles of modular forms $f$ of low filtration.

EXAMPLE 7.6. $f$ is in the kernel of $U$ and $4 \leqslant w(f) \leqslant l-1$.

Then $f$ is a low point of its $\theta$-cycle which can be expressed as follows.

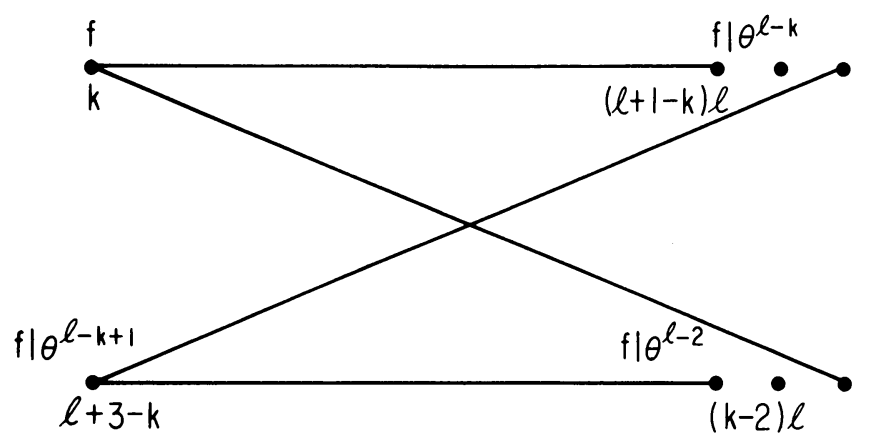

Note that in this case, the $\theta$-cycle of $f$ dips below $l+1$ in two different places as $w(f)=w\left(f \mid \theta^{l-1}\right)=k$ and also $w\left(f \mid \theta^{l-k+1}\right)=l+3-k$. Moreover, the map $\theta^{l-k+1}$ clearly gives us an isomorphism between the kernel of $U$ in weight $k$ and the kernel of $U$ in weight $l+3-k$.

COROLLARY 7.7. The kernel of $U$ is trivial for weights $l-1, l-3, l-5, l-7$ and $l-11$.

Proof. The kernel is trivial for weights 4, 6, 8, 10, and 14. Q.E.D.

EXAMPLE 7.8. $f$ is not in the kernel of $U$ and $4 \leqslant w(f) \leqslant l-1$.

We claim that under these hypotheses, $f \mid \theta$ must be a low point of its $\theta$-cycle. Indeed were this not the case, there would exist a nonzero form $g$ in the kernel of $U$ such that $w(g)=w(f)$ and such that $g|\theta=f| \theta$. By the previous corollary the filtration of $g$, and hence the filtration of $f$, cannot equal $l-1$. Therefore, $w(g-f)$ must equal $w(f)$ and, in particular, it is not divisible by $l$. But $g-f$ is in the kernel of $\theta$. This contradicts Lemma 4.1(b).

The $\theta$-cycle of $f$ can thus be represented as follows.

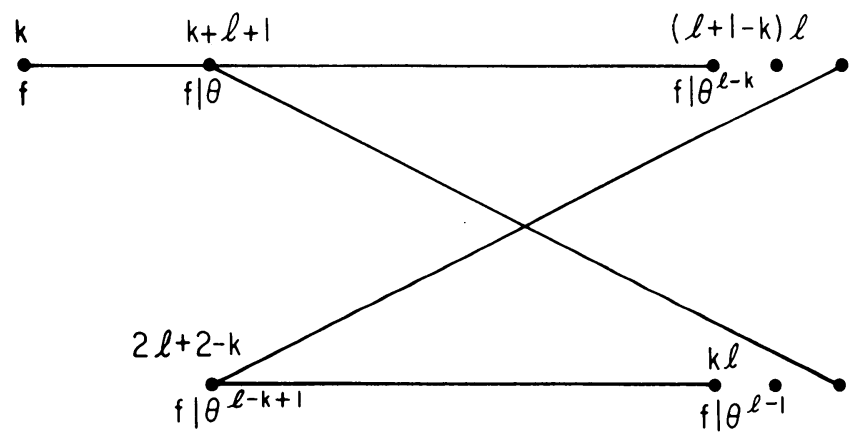


In particular, in contrast to the previous example, $f$ is outside of its $\theta$-cycle, and the $\theta$-cycle of $f$ never dips below a filtration of $l+3$.

EXAMPLE 7.9. $w(f)=l+1$.

The $\theta$-cycle of $f$, a special case of Figure 7.1, can be represented as follows.

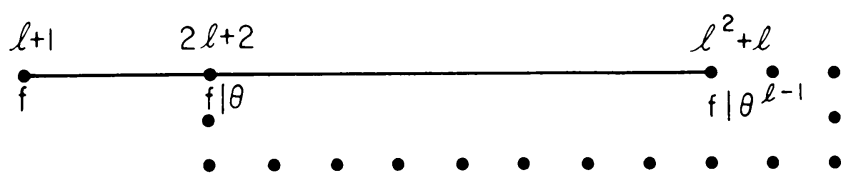

8. A better bound for the Zariski tangent dimension. Let $A_{k}$ be a $U$-nilpotent local component of $R_{k} \otimes \overline{\mathbf{F}}_{l}$. As we saw in the proof of Lemma 4.4, any simultaneous eigenvector associated to $A_{k}$ is of the form $f \mid \theta^{a}$ for some eigenvector $f$ of filtration less than or equal to $l+1$. We call $A_{k}$ an exceptional component if $f$ is of filtration exactly $l+1$.

THEOREM 8.1. If $k$ is sufficiently large, the dimension of the Zariski tangent space of all nonexceptional $U$-nilpotent components of $R_{k} \otimes \overline{\mathbf{F}}_{l}$ will be at least three.

REMARK. In fact, the conclusion of the theorem is true whenever $k \geqslant l^{3}+l^{2}$.

Proof of TheOrem 8.1. We first reduce the proof of our theorem to that of the following.

THEOREM 8.1'. If $k$ is sufficiently large, then the U-independent subrings of all nonexceptional U-nilpotent components will have Zariski tangent spaces of dimension at least two.

Reduction to Theorem 8.1'. Suppose that Theorem $8.1^{\prime}$ is true. Let $A_{j} \leftarrow A_{j+l-1} \leftarrow \ldots$ be an arbitrary nonexceptional system of $U$-nilpotent components and let $C_{j} \leftarrow C_{j+1-1} \leftarrow \ldots$ be the corresponding system of $U$-independent subrings. By hypotheses, when $k$ is sufficiently large, the Zariski tangent dimension of $C_{k}$ will be at least two. Therefore, the Zariski tangent dimension of $C=\lim C_{k}$ is at least two. Hence it follows from Theorem 6.3 that the Zariski tangent dimension of $A=\lim A_{k}$ is at least three. Using a standard argument about inverse limits which depends on the fact that the $A_{k}$ are Artin rings, we conclude that when $k$ is sufficiently large, the Zariski tangent dimension of the ring $A_{k}$ in the above surjective system will be greater than or equal to three.

Since Lemma 4.4 implies that there are only a finite number of essentially distinct surjective systems of local components, it follows that when $k$ is sufficiently large, the Zariski tangent dimension of all nonexceptional $U$-nilpotent components of $R_{k} \otimes \overline{\mathbf{F}}_{l}$ will be at least three. Q.E.D.

It thus remains only to prove Theorem $8.1^{\prime}$.

LEMMA 8.2. Let $A_{k}$ be an arbitrary $U$-nilpotent component of $R_{k} \otimes \overline{\mathbf{F}}_{l}, S_{k}$ its associated generalized eigenspace, and $C_{k} \subseteq A_{k}$ its $U$-independent subring. Suppose that the Zariski tangent dimension of $C_{k}$ is less than or equal to one. Then for any pair of forms $g, h \in S_{k}$, there exists $T \in C_{k}$ such that modulo the image of $V$ either $g \mid T=h$ or $h \mid T=g$. 
Proof. Let $\overline{S_{k}}=S_{k} /\left(S_{k} \cap\right.$ image $\left.V\right)$. Define a bilinear pairing between $\bar{S}_{k}$ and $C_{k}$ as follows: if $\bar{g} \in \bar{S}_{k}$ and $T \in C_{k}$, then $\langle\bar{g}, T\rangle=a_{1}(\bar{g} \mid T)=$ the first coefficient of the $q$-expansion of $g \mid T$. As in Lemma 6.5 one shows that this is a nondegenerate bilinear pairing.

Let $\bar{h}$ be the image in $\bar{S}_{k}$ of $h \in S_{k}$ and let $\langle\bar{h}\rangle$ be the $C_{k}$ module generated by $\bar{h}$. It is then easy to see that under the above bilinear pairing, the orthogonal complement $\langle\bar{h}\rangle^{\perp}$ equals $\left\{T \in C_{k}|h| T \in\right.$ image $\left.V\right\}$ and, in particular, is an ideal of $C_{k}$.

Since the Zariski tangent dimension of $C_{k}$ is less than or equal to one, $C_{k}$ is a principal Artin local ring. Hence any ideal of $C_{k}$ is a power of the maximal one. In particular, the set of ideals of $C_{k}$ is linearly ordered by the relation of containment.

Therefore if $g, h$ are arbitrary elements of $S_{k}$, we can assume without loss of - generality that $\langle\bar{g}\rangle^{\perp} \subseteq\langle\bar{h}\rangle^{\perp}$. But this implies that $\langle\bar{h}\rangle \subseteq\langle\bar{g}\rangle$. Q.E.D.

Lemma 8.3. Let $g, h \in S_{k}$. Suppose that there exist positive integers $b, c$ such that $w\left(g \mid \theta^{b}\right)<w\left(h \mid \theta^{b}\right)$ but $w\left(g \mid \theta^{c}\right)>w\left(h \mid \theta^{c}\right)$. Then the Zariski tangent dimension of the ring $C_{k}$ must be at least two.

Proof. Suppose that the Zariski tangent dimension of $C_{k}$ is less than or equal to one. Then by the previous lemma, there exists a $U$-independent operator $T$ such that modulo the image of $V$, either $g \mid T=h$ or $h \mid T=g$.

Assume without loss of generality that $g \mid T=h$ modulo the image of $V$. Then for any $n$ there exists a $U$-independent operator $T^{\prime}$ such that $g\left|\theta^{n}\right| T^{\prime}=h \mid \theta^{n}$. This implies that $w\left(h \mid \theta^{n}\right) \leqslant w\left(g \mid \theta^{n}\right)$ for all $n$. Q.E.D.

Proof of TheOREM 8.1'. By the above lemma, in order to complete the proof, it suffices to show that when $k$ is sufficiently large there exist $g, h \in S_{k}$ and positive integers $b, c$ such that $w\left(g \mid \theta^{b}\right)<w\left(h \mid \theta^{b}\right)$, but $w\left(g \mid \theta^{c}\right)>w\left(h \mid \theta^{c}\right)$.

Since we are in the nonexceptional case, $S_{k}$ contains a simultaneous eigenvector of the form $f \mid \theta^{a}$, where $f$ is of filtration $j$, which is strictly less than $l+1$. The remainder of the proof is divided into two cases.

Case 1. $f$ is not in the kernel of $U$.

By Example 7.8, the $\theta$-cycle of $f$ is pictorially represented as follows.

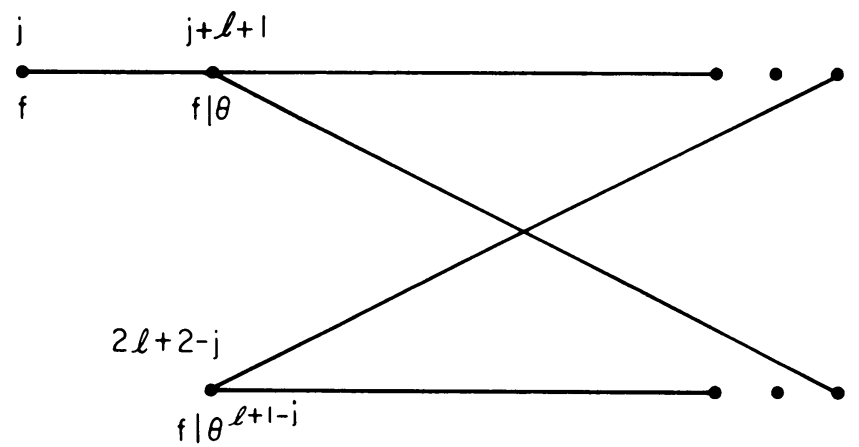

In particular, $w(f \mid \theta)=l+1+j$ and $w\left(f \mid \theta^{l+1-j}\right)=2 l+2-j$.

Thus Lemma 3.4 implies that there exists a modular form $g_{1}$ of filtration $(j+1) l+1$ in the same generalized eigenspace as $f \mid \theta$. Similarly, there exists a form $h_{1}$ of filtration $(l+2-j) l+1$ in the same generalized eigenspace as $f \mid \theta^{l+1-j}$. 
Let $g=g_{1} \mid \theta^{a-1}$ and $h=h_{1} \mid \theta^{a+j-2}$. Then if $k$ is sufficiently large, both $g$ and $h$ will be in $S_{k}$, the generalized eigenspace corresponding to $f \mid \theta^{a}$.

But

$$
\begin{aligned}
w\left(g \mid \theta^{l+1-a}\right) & =w\left(g_{1} \mid \theta\right)=(j+2) l+2 \\
& <(l+2) l+j+1=w\left(h_{1} \mid \theta^{j}\right)=w\left(h \mid \theta^{l+1-a}\right),
\end{aligned}
$$

and, on the other hand,

$$
\begin{aligned}
w\left(g \mid \theta^{l+2-a-j}\right) & =w\left(g_{1} \mid \theta^{l+1-j}\right)=(l+3) l+2-j \\
& >(l+3-j) l+2=w\left(h_{1} \mid \theta\right)=w\left(h \mid \theta^{l+2-a-j}\right),
\end{aligned}
$$

which completes the proof in this case.

The idea behind this proof is illustrated by the following diagram.

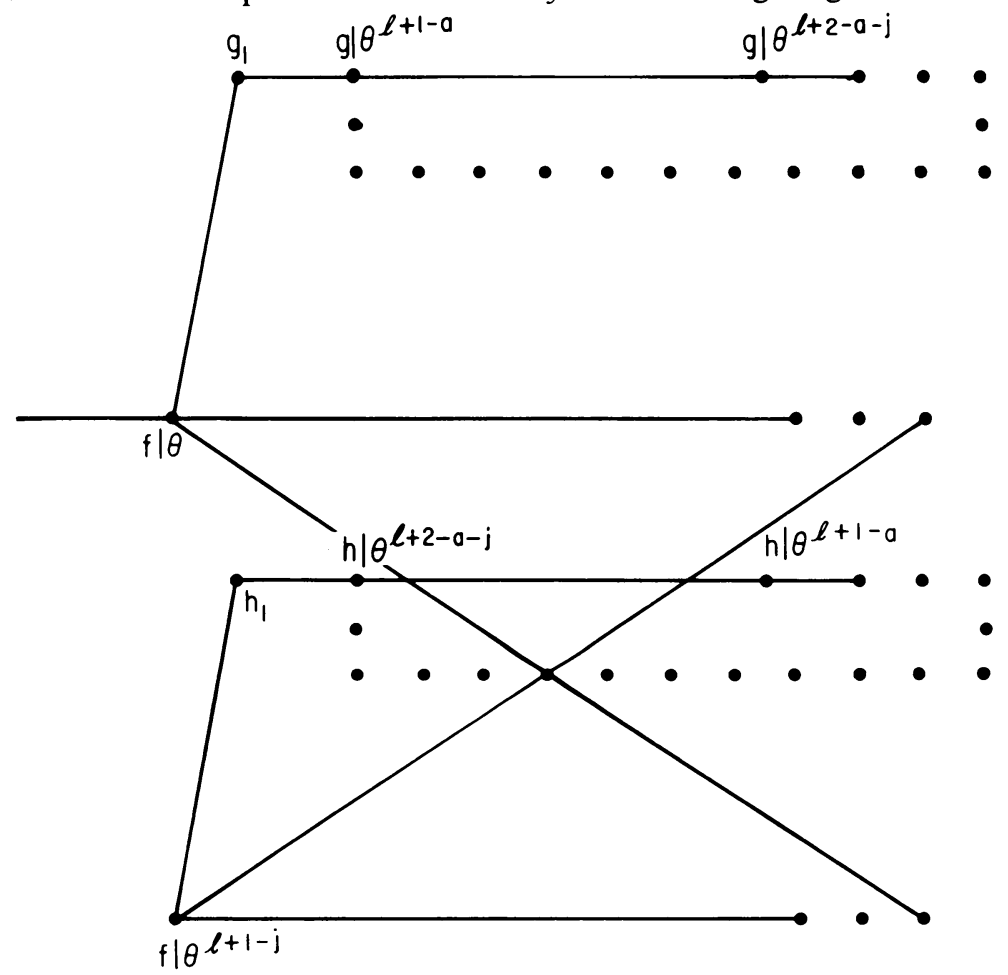

Case 2. $f$ is in the kernel of $U$.

The proof is analogous to that of Case 1, only the numbers are slightly different. By Example 7.6, the $\theta$-cycle of $f$ can be represented as follows.

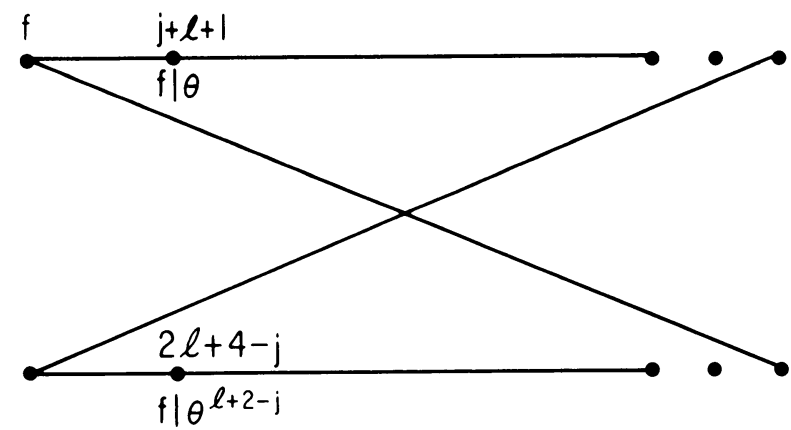


In particular, $w(f \mid \theta)=l+1+j$ and $w\left(f \mid \theta^{l+2-j}\right)=2 l+4-j$.

Again Lemma 3.4 implies that there exists a $g_{1}$ of filtration $(j+1) l+1$ in the same generalized eigenspace as $f \mid \theta$ and a form $h_{1}$ of filtration $(l+4-j) l+1$ in the same generalized eigenspace as $f \mid \theta^{l+2-j}$.

When $k$ is sufficiently large, the forms $g=g_{1} \mid \theta^{a-1}$ and $h=h_{1} \mid \theta^{a+j-3}$ will belong to the generalized eigenspace $S_{k}$.

However,

$$
\begin{aligned}
w\left(g \mid \theta^{l+1-a}\right) & =w\left(g_{1} \mid \theta\right)=(j+2) l+2<(l+3) l+j \\
& =w\left(h_{1} \mid \theta^{j-1}\right)=w\left(h \mid \theta^{l+1-a}\right),
\end{aligned}
$$

and, on the other hand,

$$
\begin{aligned}
w\left(g \mid \theta^{l+3-j-a}\right) & =w\left(g_{1} \mid \theta^{l+2-j}\right)=(l+4) l+3-j \\
& >(l+5-j) l+2=w\left(h_{1} \mid \theta\right)=w\left(h \mid \theta^{l+3-j-a}\right) . \quad \text { Q.E.D. }
\end{aligned}
$$

REMARK. The above proof fails in the exceptional case because we cannot find a simultaneous eigenvector in $S_{k}$ whose $\theta$-cycle has two low points, i.e.:

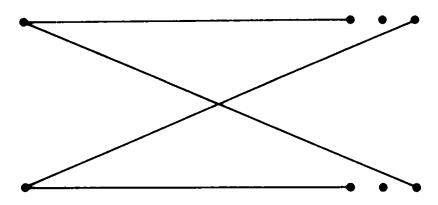

In the exceptional case, the $\theta$ cycle of a simultaneous eigenvector has only one low point, i.e.:

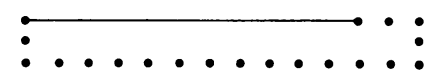

\section{REFERENCES}

1. N. Jochnowitz, The index of the Hecke ring $\mathbf{T}_{k}$ in the ring of integers of $\mathbf{T}_{k} \otimes \mathbf{Q}$, Duke Math. J. 46 (1979), 861-869.

2. Congruences between systems of eigenvalues of modular forms, Trans. Amer. Math. Soc. 270 (1982), 269-285.

3. N. Katz, p-adic properties of modular schemes and modular forms, Lecture Notes in Math., vol. 350, Springer-Verlag, Berlin and New York, 1973, pp. 69-190.

4. __ A result on modular forms in characteristic $p$, Lecture Notes in Math., vol. 601, Springer-Verlag, Berlin and New York, 1976, pp. 53-56.

5. V. Miller, Diophantine and p-adic analysis of elliptic curves and modular forms, Ph. D. Thesis, Harvard, June, 1975.

6. J.-P. Serre, Congruences et formes modulaires (d'apres Swinnerton-Dyer), Sém. Bourbaki, Exp. 416, Lecture Notes in Math., vol. 317, Springer-Verlag, Berlin and New York, 1973, pp. 319-338.

7. Formes modulaires et fonctions zêta p-adiques, Lecture Notes in Math., vol. 350, SpringerVerlag, Berlin and New York, 1973, pp. 191-268.

8. H. P. F. Swinnerton-Dyer, On l-adic representations and congruences for coefficients of modular forms, Lecture Notes in Math., vol. 350, Springer-Verlag, Berlin and New York, 1973, pp. 1-55.

Department of Mathematics, Brown University, Providence, Rhode Island 02912 